\title{
ULTRASIM: Ultrasound in trauma simulation
}

\author{
Devon McLean, BSc*; Leanne Hewitson, $\mathrm{MD}^{\dagger}$; Paul Atkinson, $\mathrm{MB}^{*}$; ; David Lewis, $\mathrm{BM}^{* *}$;
} Jacqueline Fraser, $\mathrm{BN}^{\ddagger}$; Glenn Verheul, $\mathrm{MD}^{\text {\%; }}$ Jayanand Mekwan, $\mathrm{MB}^{* *}$; Bryn Robinson, $\mathrm{PhD}^{* *}$

\begin{abstract}
CLINICIAN'S CAPSULE
What is known about the topic?

Ultrasound simulation is an invaluable resource in medical education. However, the equipment required is often prohibitively expensive.

What did this study ask?

Does the use of a low-cost ultrasound simulator in trauma simulation improve diagnostic capabilities?

What did this study find?

Study participants experienced improvements in diagnostic accuracy, confidence, and precision following the use of this ultrasound simulator.

Why does this study matter to clinicians?

This low-cost ultrasound simulator provides an alternative to current technology and can easily be implemented into medical simulations.
\end{abstract}

\section{ABSTRACT}

Objective: This study sought to assess the effectiveness of ultrasound simulation as a component of high-fidelity trauma simulation, in training diagnostic capabilities of resident and attending physicians participating in simulated trauma scenarios. Methods: Twelve residents and 20 attending physicians participated in 114 trauma simulations. Participants generated a ranked differential diagnosis list after a physical exam and subsequently after a simulated extended focused assessment with sonography for trauma (E-FAST) ultrasound scan. We compared reports to determine whether the addition of ultrasound improved diagnostic performance.

Results: The primary diagnosis accuracy improved significantly with the addition of simulated ultrasound $(p<0.0001)$. Median diagnostic ranking scores also improved $(p<0.0001)$. Further, participants reported a higher confidence in their diagnoses $(p<0.0001)$ and narrowed their differential diagnosis list $(p<0.0001)$.

Conclusion: We demonstrated that a low-cost ultrasound simulator can be successfully integrated into trauma simulations, resulting in an associated improvement in measures of diagnostic accuracy, confidence, and precision for participating resident and attending physicians.

\section{RÉSUMÉ}

Objectif: L'essai visait à évaluer l'efficacité des simulations d'échographie comme moyen de représentation réaliste de traumas, dans l'amélioration de la compétence diagnostique de résidents et de médecins traitants qui avaient participé à des études de cas factices de trauma.

Méthode: $\mathrm{Au}$ total, 12 résidents et 20 médecins traitants ont participé à 114 simulations de trauma. Les sujets ont dressé une liste ordonnée de diagnostics différentiels après un examen physique et une autre après une échographie simulée E-FAST. II y a ensuite eu comparaison des rapports afin de déterminer si l'ajout de l'échographie avait permis d'améliorer la performance relative à l'exactitude diagnostique.

Résultats: L'exactitude diagnostique initiale s'est améliorée de manière significative avec l'ajout des simulations d'échographie $(p<0,0001)$. Il y a également eu une amélioration des résultats médians relatifs au classement des diagnostics $(p<0,0001)$. En outre, les participants ont fait état d'un plus grand degré de confiance personnelle dans l'exactitude diagnostique $(p<0,0001)$ et ont raccourci leur liste de diagnostics différentiels $(p<0,0001)$.

Conclusions: Les résultats de l'étude démontrent que l'utilisation d'un simulateur d'échographie à bas coût peut donner de bons résultats dans l'étude de cas factices de trauma; en effet, une amélioration des mesures de l'exactitude et de la précision diagnostiques ainsi que de la confiance personnelle a été observée chez les résidents et les médecins traitants qui avaient participé à des séances de formation.

Keywords: education, point-of-care ultrasound, simulation, trauma

\section{INTRODUCTION}

In trauma and critical care, clinicians are required to assess and initiate treatment for time-critical, often life-threatening injuries. One of the most versatile approaches used in this setting is ultrasonography.

From the *Dalhousie Medicine New Brunswick, Saint John, NB; †Vernon Jubilee Hospital, Vernon, BC; $¥$ Department of Emergency Medicine, Saint John Regional Hospital, Saint John, NB; §New Brunswick Trauma Program, Saint John, NB; IDepartment of Emergency Medicine, Calvary Health Care, Canberra, Australia; and the ${ }^{* *}$ Maritime SPOR SUPPORT Unit, Saint John, NB.

Correspondence to: Devon McLean, 215 Twin Oaks Drive, Moncton, NB E1G 4W4; Email: devonmclean@live.ca 
Point-of-care ultrasound (POCUS), an ultrasound exam performed and interpreted at the bedside by a primary care provider, ${ }^{1}$ is invaluable in rapidly diagnosing patients presenting with trauma. ${ }^{2}$ Perhaps one of the most widely used POCUS scanning protocols in this setting is the extended focused assessment with sonography for trauma (E-FAST), which includes views into the abdomen, pelvis, and chest to assess for evidence of hemoperitoneum, hemothorax, or pneumothorax. $^{3}$

High-fidelity simulation, which makes use of technologically advanced mannequins in simulations that mimic clinic practice, is becoming increasingly popular in medical education. ${ }^{4}$ Further, hybrid simulation, which integrates POCUS into high-fidelity simulation, can lead to higher satisfaction amongst learners, and increase the overall fidelity of the simulation. ${ }^{4}$ The ultrasound simulator used in this study, the emergency department ultrasound simulator ${ }^{5}$ (edus2), is an example of a low-cost device that allows learners to perform real-time scans during high-fidelity simulation. ${ }^{6}$

Equipment for medical simulation can be prohibitively expensive. This study looked at whether the addition of a low-cost ultrasound simulator to highfidelity trauma simulation was associated with an improvement in diagnostic accuracy, confidence, and precision.

\section{METHODS}

This prospective observational study was conducted at an urban regional hospital. The population consisted of resident (PGY1-3) and attending physicians. This study was approved by the Horizon Health Network Research Ethics Board.

All participants received standardized didactic and hands-on-training with the edus2. This simulator consists of radiofrequency identification chips tagged with pre-recorded videos of ultrasound scans, which are placed under the skin of a Laerdal SimMan 3G mannequin. When a simulated ultrasound probe is passed over the chips, the video is played on an adjacent screen (Supplementary Appendix 1).

Participants were given a brief case presentation and performed a history and physical exam of the mannequin. From this, they generated a list of up to five possible diagnoses with corresponding confidence (from $1 \%$ to $100 \%$ ) that a given diagnosis matched the correct pathology. Participants then completed an E-FAST scan using the edus 2 and subsequently recorded a new differential diagnosis list.

Following testing, a brief questionnaire regarding perceptions of realism and educational utility of the edus2 was completed by each participant (Supplementary Appendix 2).

\section{RESULTS}

Twelve residents and 20 attending physicians completed up to 6 simulations each, for a total of 114 scenarios. Table 1 presents the descriptive statistics for both residents and attending physicians on frequency of correct primary diagnoses, diagnostic accuracy, confidence, and precision, both before and after using the edus2.

The diagnostic accuracy was assessed by comparing the total of number of correct primary diagnoses before and

\begin{tabular}{|c|c|c|c|c|}
\hline & & $\begin{array}{l}\text { Overall } \\
(n=114)\end{array}$ & $\begin{array}{l}\text { Residents } \\
(n=72)\end{array}$ & $\begin{array}{l}\text { Attending physicians } \\
\qquad(n=42)\end{array}$ \\
\hline \multirow[t]{3}{*}{ Diagnostic accuracy (\%) } & Pre-POCUS & $46 \%$ & $43 \%$ & $52 \%$ \\
\hline & Post-POCUS & $85 \%$ & $89 \%$ & $79 \%$ \\
\hline & $p$ value & $p<0.0001$ & $p<0.0001$ & $p=0.0116$ \\
\hline \multirow[t]{3}{*}{ Median rank $(/ Q R)$} & Pre-POCUS & $3.8(3.0-4.9)$ & $4.0(3.2-4.0)$ & $3.9(2.6-5.0)$ \\
\hline & Post-POCUS & $5.0(4.7-5.0)$ & $4.8(4.7-5.0)$ & $5.0(4.2-5.0)$ \\
\hline & $p$ value & $p<0.0001$ & $p=0.001$ & $p=0.0098$ \\
\hline \multirow[t]{3}{*}{ Mean confidence score (SD) } & Pre-POCUS & $53.1(22.8)$ & $47.6(13.6)$ & $56.5(26.7)$ \\
\hline & Post-POCUS & $83.5(19.1)$ & $83.6(7.0)$ & $83.5(23.9)$ \\
\hline & $p$ value & $p<0.0001$ & $p<0.0001$ & $p<0.0001$ \\
\hline \multirow[t]{3}{*}{ Median precision score $(/ Q R)$} & Pre-POCUS & $3.5(2.9-4.4)$ & $3.2(2.8-4.3)$ & $4.0(3.1-4.4)$ \\
\hline & Post-POCUS & $2.4(1.9-3.0)$ & $2.4(1.7-2.8)$ & $2.0(2.0-3.3)$ \\
\hline & $p$ value & $p<0.0001$ & $p<0.0001$ & $p<0.0001$ \\
\hline
\end{tabular}


after the ultrasound. Following the use of the edus2, participants were more likely to rank the correct diagnosis at the top of their differential list $(p<0.0001)$. This improvement was seen for residents $(p<0.0001)$ and attending physicians $(p=0.0116)$.

For a diagnostic rank score, if participants ranked the correct diagnosis as their first choice, they received five points; if second, they received four points, and so on. Following an ultrasound, it was more likely that participants would rank the correct diagnosis higher on their differential diagnosis lists $(p<0.0001)$ (Supplementary Appendix 3). The same finding was true for resident $(p=0.001)$ and attending physicians $(p=0.0098)$.

The diagnostic confidence was defined as the percent confidence (from $1 \%$ to $100 \%$ ) indicated with the correct diagnosis. Overall, participants reported higher percentages of confidence in their diagnoses following an ultrasound $(p<0.0001)$ (Supplementary Appendix 4). Specifically, residents' $(p<0.0001)$ and attending physicians' $(p<0.0001)$ confidence also increased.

The diagnostic precision refers to the size of the differential diagnosis list, with less items considered more precise. Overall, participants narrowed their differential diagnosis list following POCUS $(p<0.0001)$ (Supplementary Appendix 5). Again, this reduction was significant in both residents $(p<0.0001)$ and attending physicians $(p<0.0001)$.

Lastly, on the questionnaire, participants rated the realism and the potential impact of the edus2 future medical education to be very high (median rating of 4 out of 5 for all items assessed).

\section{DISCUSSION}

We have found that there were statistically significant increases in the number of correct primary diagnoses made, diagnostic rank score, confidence in the correct diagnosis, and diagnostic precision following the use of an ultrasound simulator.

Recent studies have found similar improvements in diagnostic capabilities following POCUS. Parks et al. ${ }^{7}$ found an improvement in medical learner's diagnostic accuracy, confidence, and precision when an ultrasound was introduced into simulated cardiorespiratory scenarios. Similarly, in the clinical setting, Jones et al. found that physicians improved their diagnostic precision and proportion of correct primary diagnoses after using ultrasound in patients presenting with undifferentiated hypotension. ${ }^{8}$
One limitation of this study is that the edus 2 simulator requires users to pass the scanner only near the locations of the E-FAST scan to generate a perfect image. It does not simulate body habitus or variations in anatomy. In addition, without a control group, we can report on associated improvements in diagnostic capabilities but cannot suggest causation.

\section{CONCLUSIONS}

Given the ever-increasing cost of medical simulation equipment, simulators such as the edus 2 are important low-cost alternatives. One study has shown that this simulator costs less than CAD $\$ 150$ to assemble. ${ }^{9}$ Considering the cost, ease of use, and associated improved diagnostic capabilities, the edus 2 could easily be integrated into simulation programs across the country. In the future, we hope to compare the edus 2 to other high-fidelity ultrasound simulators to assess for differences in impact on diagnostic capabilities, or in user perception of educational utility.

Acknowledgements: We thank all study participants for volunteering their time to contribute to this study. Additionally, we thank Susan Benjamin and the New Brunswick Trauma Program for their continued guidance and support.

Competing interests: None declared. This study received funding from the New Brunswick Trauma Program (2013) and the Health Innovation Research Fund (2013).

\section{SUPPLEMENTARY MATERIAL}

To view supplementary material for this article, please visit https://doi.org/10.1017/cem.2018.56

\section{REFERENCES}

1. Atkinson P, Ross P, Henneberry R. Coming of age: emergency point of care ultrasonography in Canada. CFEM 2014;16(4):265-8.

2. Boulanger BR, McLellan BA, Brenneman FD, et al. Prospective evidence of the superiority of a sonographybased algorithm in the assessment of blunt abdominal injury. 7 Trauma $1999 ; 47(4): 632-7$.

3. Williams SR, Perera P, Gharahbaghian L. The FAST and E-FAST in 2013: trauma ultrasonography: overview, practical techniques, controversies, and new frontiers. Crit Care Clin 2014;30(1):119-50, vi, doi:10.1016/j.ccc.2013.08.005.

4. Girzadas DV Jr, Antonis MS, Zerth H, et al. Hybrid simulation combining a high fidelity scenario with a pelvic ultrasound task trainer enhances the training and evaluation 
of endovaginal ultrasound skills. Acad Emerg Med 2009; 16(5):429-35; doi:10.1111/j.1553-2712.2009.00399.x.

5. Paul Kulyk PO. Emergency department ultrasound simulator. Available at: http://www.edus2.com/ (accessed 24 June 2016).

6. Olszynski PA, Harris T, Renihan P, et al. Ultrasound during critical care simulation: a randomized crossover study. C7EM 2016;18(3):183-90; doi:10.1017/cem.2015.87.

7. Parks AR, Verheul G, Leblanc-Duchin D, Atkinson P. Effect of a point-of-care ultrasound protocol on the diagnostic performance of medical learners during simulated cardiorespiratory scenarios. CFEM 2015;17(3):263-9; doi:10.1017/cem.2014.41.

8. Jones AE, Tayal VS, Sullivan DM, Kline JA. Randomized, controlled trial of immediate versus delayed goal-directed ultrasound to identify the cause of nontraumatic hypotension in emergency department patients. Crit Care Med 2004;32 (8):1703-8; doi:10.1097/01.CCM.0000133017.34137.82.

9. Damjonovic D, Goebel U, Fischer B, et al. An easy-to-build, low-budget point-of-care ultrasound simulator: from Linux to a web-based solution. Crit Ultrasound 7 2017;9:4. doi:10.1186/s13089-017-0061-4. 